\title{
Relativistic Scattering States of the Hellmann Potential
}

\author{
B.H. YazARloO ${ }^{a, *}$, H. MehrabaN ${ }^{a}$ And H. HASSANABADi ${ }^{b}$ \\ ${ }^{a}$ Physics Department, Semnan University, P.O. Box 35195-363, Semnan, Iran \\ ${ }^{b}$ Physics Department, Shahrood University, Shahrood, Iran \\ (Received August 21, 2014; revised version January 15, 2015; in final form January 23, 2015)
}

\begin{abstract}
In this letter, the scattering state solutions of the Dirac equation for spin and pseudospin symmetries are obtained for the Hellmann potential. The normalized wave functions and scattering phase shifts are calculated for both spin and pseudospin symmetries. Scattering properties for Coulomb-like and Yukawa-like potentials are also studied as limiting cases.
\end{abstract}

DOI: 10.12693/APhysPolA.127.684

PACS: 03.65.Nk, 03.65.Pm

\section{Introduction}

The Dirac equation [1] has become the most appealing relativistic wave equation in the field of mathematical physics for spin- $1 / 2$ particles. One of the interesting problems in the Dirac equation is the concept of spin and pseudospin symmetries. These symmetries of the Dirac Hamiltonian were discovered many years ago. However, the spin and pseudospin symmetries have been recently recognized empirically in nuclear and hadronic spectroscopies. The solution of the Dirac equation with mixed potentials for particles such as atoms, nuclei and hadrons play a central role in a realistic nuclear system. In order to investigate the nuclear shell structure, the study of the pseudospin and spin symmetric solutions of the Dirac equation has been an important area of research in nuclear physics. However, spin symmetry is relevant to mesons [2], and the pseudospin symmetry refers to a quasi-degeneracy of single nucleon doublets $[3,4]$. Recently, many works have been done to solve the Dirac equation so to obtain the energy equation and the twocomponent spinor wave functions [5-18]. In the recent years, investigation of scattering states properties of nonrelativistic and relativistic wave equations in quantum mechanics has been in great attention. In order to understand the studied quantum system completely, we should study the both bound states and the scattering states for a given quantum system.

In the present work, we deal with the solution of scattering state of the Dirac equation for spin and pseudospin symmetries with the Hellmann potential which is the superposition of the Coulomb and Yukawa potentials and defined as [19]:

$$
v(r)=-\frac{a}{r}+b \frac{\mathrm{e}^{-\alpha r}}{r}
$$

where $a$ and $b$ are the strengths of the Coulomb and the Yukawa potentials, respectively, and $\alpha$ is the screening parameter. This potential has many applications in atomic physics and condensed matter physics [20-22].

${ }^{*}$ corresponding author; e-mail: hoda.yazarloo@gmail.com
In what follows, we first review the Dirac equation and present scattering state solution of Dirac equation in the presence of spin and pseudo-spin symmetries in Sect. 3, and discussed some special cases of Hellmann potential in Sect. 4. Finally our conclusion appeared in Sect. 5.

\section{Theory of Dirac equation with tensor coupling}

The Dirac equation for spin- $\frac{1}{2}$ particles moving in an attractive scalar potential $S(r)$, a repulsive vector potential $V(r)$ and a tensor potential $U(r)$ in the relativistic unit $(\hbar=c=1)$ is [23]:

$$
\begin{aligned}
& {[\boldsymbol{\alpha} \cdot \boldsymbol{p}+\beta(M+S(r))-\mathrm{i} \beta \boldsymbol{\alpha} \cdot \hat{r} U(r)] \psi(r)=} \\
& \quad[E-V(r)] \psi(r),
\end{aligned}
$$

where $E$ is the relativistic energy of the system, $\boldsymbol{p}=-\mathrm{i} \boldsymbol{\nabla}$ is the three-dimensional momentum operator and $M$ is the mass of the fermionic particle. $\boldsymbol{\alpha}, \beta$ are the $4 \times 4$ Dirac matrices given as

$$
\boldsymbol{\alpha}=\left(\begin{array}{cc}
0 & \boldsymbol{\sigma}_{i} \\
\boldsymbol{\sigma}_{i} & 0
\end{array}\right), \beta=\left(\begin{array}{cc}
I & 0 \\
0 & -I
\end{array}\right),
$$

where $I$ is $2 \times 2$ unitary matrix and $\sigma_{i}$ are the Pauli threevector matrices. The eigenvalues of the spin-orbit coupling operator are $\kappa=\left(j+\frac{1}{2}\right) \succ 0, \kappa=-\left(j+\frac{1}{2}\right) \prec 0$ for unaligned $j=l-\frac{1}{2}$ and the aligned spin $j=l+\frac{1}{2}$ respectively. The set $\left(H, K, J^{2}, J_{z}\right)$ forms a complete set of conserved quantities. Thus, we can write the spinors as [20]:

$$
\psi_{n \kappa}(r)=\frac{1}{r}\left(\begin{array}{l}
F_{n \kappa}(r) Y_{j m}^{l}(\theta, \varphi) \\
i G_{n \kappa}(r) Y_{j m}^{\tilde{l}}(\theta, \varphi)
\end{array}\right),
$$

where $F_{n \kappa}(r), G_{n \kappa}(r)$ represent the upper and lower components of the Dirac spinors. $Y_{j m}^{l}(\theta, \phi), Y_{j m}^{\tilde{l}}(\theta, \phi)$ are the spin and pseudospin spherical harmonics and $m$ is the projection on the $z$-axis. By using Eq. (1) and Eq. (4) we find the following two coupled first-order Dirac equations:

$$
\begin{aligned}
& \left(\frac{\mathrm{d}}{\mathrm{d} r}+\frac{\kappa}{r}\right) F_{n \kappa}(r)=\left(M+E_{n \kappa}-\Delta(r)\right) G_{n \kappa}(r), \\
& \left(\frac{\mathrm{d}}{\mathrm{d} r}-\frac{\kappa}{r}\right) G_{n \kappa}(r)=\left(M-E_{n \kappa}+\Sigma(r)\right) F_{n \kappa}(r),
\end{aligned}
$$

and the extension to tensor interaction becomes 


$$
\begin{gathered}
\left(\frac{\mathrm{d}}{\mathrm{d} r}+\frac{\kappa}{r}-U(r)\right) F_{n \kappa}(r)= \\
\left(M+E_{n \kappa}-\Delta(r)\right) G_{n \kappa}(r), \\
\left(\frac{\mathrm{d}}{\mathrm{d} r}-\frac{\kappa}{r}+U(r)\right) G_{n \kappa}(r)= \\
\left(M-E_{n \kappa}+\Sigma(r)\right) F_{n \kappa}(r),
\end{gathered}
$$

where

$$
\begin{aligned}
& \Delta(r)=V(r)-S(r), \\
& \Sigma(r)=V(r)+S(r) .
\end{aligned}
$$

Eliminating $F_{n \kappa}(r)$ and $G_{n \kappa}(r)$ in Eqs. (7) and (8), we obtain the second-order Schrödinger-like equation as

$$
\begin{gathered}
{\left[\frac{\mathrm{d}^{2}}{\mathrm{~d} r^{2}}-\frac{\kappa(\kappa+1)}{r^{2}}+\frac{2 \kappa U(r)}{r}-\frac{\mathrm{d} U(r)}{\mathrm{d} r}-U^{2}(r)\right.} \\
\quad-\left(M+E_{n \kappa}-\Delta(r)\right)\left(M-E_{n \kappa}+\Sigma(r)\right) \\
\left.+\frac{\frac{\mathrm{d} \Delta(r)}{\mathrm{d} r}\left(\frac{\mathrm{d}}{\mathrm{d} r}+\frac{\kappa}{r}-U(r)\right)}{\left(M+E_{n \kappa}-\Delta(r)\right)}\right] F_{n \kappa}(r)=0, \\
{\left[\frac{\mathrm{d}^{2}}{\mathrm{~d} r^{2}}-\frac{\kappa(\kappa-1)}{r^{2}}+\frac{2 \kappa U(r)}{r}+\frac{\mathrm{d} U(r)}{\mathrm{d} r}-U^{2}(r)\right.} \\
\quad-\left(M+E_{n \kappa}-\Delta(r)\right)\left(M-E_{n \kappa}+\Sigma(r)\right)+ \\
\left.\frac{\frac{\mathrm{d} \Sigma(r)}{\mathrm{d} r}\left(\frac{\mathrm{d}}{\mathrm{d} r}-\frac{\kappa}{r}+U(r)\right)}{\left(M+E_{n \kappa}-\Sigma(r)\right)}\right] G_{n \kappa}(r)=0,
\end{gathered}
$$

where $\kappa(\kappa-1)=\tilde{l}(\tilde{l}+1), \kappa(\kappa+1)=l(l+1)$.

\section{Scattering states of pseudospin and spin symmetry limits}

In the following section, we intend to investigate the scattering states of the Dirac equation with the Hellmann potential.

\subsection{Pseudospin symmetry limit}

The pseudospin symmetry occurs in the Dirac theory as $\frac{\mathrm{d} \Sigma(r)}{\mathrm{d} r}=0$ or equivalently $\Sigma(r)=C_{p s}=$ const. In order to find scattering solution of the Dirac equation under the pseudospin symmetry limit, we take the difference of the scalar and vector potentials as the Hellmann potential

$$
\Delta(r)=-\frac{a^{p s}}{r}+b^{p s} \frac{\mathrm{e}^{-\alpha r}}{r},
$$

and the tensor interaction has the following form:

$$
U(r)=-\frac{H}{r}
$$

Substituting Eqs. (13) and (14) into Eq. (12) yields

$$
\begin{aligned}
& {\left[\frac{\mathrm{d}^{2}}{\mathrm{~d} r^{2}}-\alpha^{2} \eta_{\kappa}\left(\eta_{\kappa}-1\right) \frac{1}{\left(1-\mathrm{e}^{-\alpha r}\right)^{2}}\right.} \\
& \quad-a^{p s} \alpha\left(M-E_{n, \kappa}^{p s}+C_{p s}\right) \frac{1}{\left(1-\mathrm{e}^{-\alpha r}\right)} \\
& \quad+b^{p s} \alpha\left(M-E_{n, \kappa}^{p s}+C_{p s}\right) \frac{\mathrm{e}^{-\alpha r}}{1-\mathrm{e}^{-\alpha r}} \\
& \left.\quad-\left(M+E_{n, \kappa}^{p s}\right)\left(M-E_{n, \kappa}^{p s}+C_{p s}\right)\right] G_{n, \kappa}^{p s}(r)=0,(15)
\end{aligned}
$$

where we make use of the following approximation $[24,25]$ :

$$
\frac{1}{r^{2}} \approx \frac{\alpha^{2}}{\left(1-\mathrm{e}^{-\alpha r}\right)^{2}} .
$$

Using a change of variable of the form $s=1-\mathrm{e}^{-\alpha r}$, Eq. (15) can be written as

$$
\begin{aligned}
& {\left[s(1-s) \frac{\mathrm{d}^{2}}{\mathrm{~d} s^{2}}-s \frac{\mathrm{d}}{\mathrm{d} s}-\eta_{\kappa}\left(\eta_{\kappa}-1\right) \frac{1}{s(1-s)}\right.} \\
& \quad-\frac{1}{\alpha^{2}}\left(M+E_{n, \kappa}^{p s}\right)\left(M-E_{n, \kappa}^{p s}+C_{p s}\right) \frac{s}{1-s} \\
& \quad-\frac{a^{p s}}{\alpha}\left(M-E_{n, \kappa}^{p s}+C_{p s}\right) \frac{1}{1-s} \\
& \left.\quad+\frac{b^{p s}}{\alpha}\left(M-E_{n, \kappa}^{p s}+C_{p s}\right)\right] G_{n \kappa}^{p s}(s)=0 .
\end{aligned}
$$

Introducing $G_{n \kappa}^{p s}(s)=s^{\gamma}(1-s)^{\beta} g_{n \kappa}^{p s}(s)$ and substituting it into Eq. (17), we obtain

$$
\begin{gathered}
{\left[s(1-s) \frac{\mathrm{d}^{2}}{\mathrm{~d} s^{2}}+(2 \gamma-(1+2 \gamma+2 \beta) s) \frac{\mathrm{d}}{\mathrm{d} s}\right.} \\
\left.-\left(\gamma^{2}+\beta^{2}+2 \beta \gamma-\Lambda_{3}\right)\right] g_{n \kappa}^{p s}(s)=0,
\end{gathered}
$$

where

$$
\begin{aligned}
& \gamma=\eta_{\kappa}, \\
& \beta=-\frac{i k}{\alpha}, \quad k=\sqrt{\alpha^{2} \Lambda_{2}}
\end{aligned}
$$

and

$$
\begin{aligned}
\Lambda_{1} & =-\eta_{\kappa}\left(\eta_{\kappa}-1\right), \\
\Lambda_{2} & =-\eta_{\kappa}\left(\eta_{\kappa}-1\right)-\frac{1}{\alpha^{2}}\left(M+E_{n, \kappa}^{p s}\right) \\
& \times\left(M-E_{n, \kappa}^{p s}+C_{p s}\right)-\frac{a^{p s}}{\alpha}\left(M-E_{n, \kappa}^{p s}+C_{p s}\right), \\
\Lambda_{3} & =\frac{1}{\alpha^{2}}\left(M+E_{n, \kappa}^{p s}\right)\left(M-E_{n, \kappa}^{p s}+C_{p s}\right) \\
& +\frac{b^{p s}}{\alpha}\left(M-E_{n, \kappa}^{p s}+C_{p s}\right),
\end{aligned}
$$

If we take

$$
\begin{aligned}
& \chi_{1}=\gamma+\beta+\sqrt{\Lambda_{3}}, \\
& \chi_{2}=\gamma+\beta-\sqrt{\Lambda_{3}}, \\
& \chi_{3}=2 \gamma,
\end{aligned}
$$

Eq. (17) satisfies the hypergeometric equation

$$
\begin{aligned}
& {\left[s(1-s) \frac{\mathrm{d}^{2}}{\mathrm{~d} s^{2}}+\left(\chi_{3}-\left(1+\chi_{1}+\chi_{2}\right) s\right) \frac{\mathrm{d}}{\mathrm{d} s}-\chi_{1} \chi_{2}\right]} \\
& \quad \times g_{n \kappa}^{p s}(s)=0,
\end{aligned}
$$

where

$$
g_{n \kappa}^{p s}(s)={ }_{2} F_{1}\left(\chi_{1}, \chi_{2}, \chi_{3} ; s\right) .
$$

Therefore, we may write down the radial wave functions as

$$
\begin{gathered}
G_{n \kappa}^{p s}(r)=N_{n \kappa}^{p s} \mathrm{e}^{\mathrm{i} k r}\left(1-\mathrm{e}^{-\alpha r}\right)^{\eta_{\kappa}} \\
\times{ }_{2} F_{1}\left(\chi_{1}, \chi_{2}, \chi_{3} ; 1-\mathrm{e}^{-\alpha r}\right) .
\end{gathered}
$$

Let us study to calculate the normalization constant and the phase shifts. Here, we recall the following properties of hypergeometric function: 


$$
\begin{aligned}
& { }_{2} F_{1}\left(\chi_{1}, \chi_{2}, \chi_{3} ; 0\right)=1 \\
& { }_{2} F_{1}\left(\chi_{1}, \chi_{2}, \chi_{3} ; s\right)=\frac{\Gamma\left(\chi_{3}\right) \Gamma\left(\chi_{3}-\chi_{1}-\chi_{2}\right)}{\Gamma\left(\chi_{3}-\chi_{1}\right) \Gamma\left(\chi_{3}-\chi_{2}\right)} \\
& \times_{2} F_{1}\left(\chi_{1}, \chi_{2}, \chi_{1}+\chi_{2}-\chi_{3}+1 ; 1-s\right) \\
& +(1-s)^{\chi_{3}-\chi_{1}-\chi_{2}} \frac{\Gamma\left(\chi_{3}\right) \Gamma\left(\chi_{1}+\chi_{2}-\chi_{3}\right)}{\Gamma\left(\chi_{1}\right) \Gamma\left(\chi_{2}\right)} \\
& \times_{2} F_{1}\left(\chi_{3}-\chi_{1}, \chi_{3}-\chi_{2}, \chi_{3}-\chi_{1}-\chi_{2}+1 ; 1-s\right) \text {. }
\end{aligned}
$$

From the properties of the hypergeometric functions ${ }_{2} F_{1}\left(\chi_{1}, \chi_{2}, \chi_{3} ; 1-\mathrm{e}^{-\alpha r}\right)$ for $r \rightarrow \infty(s \rightarrow 1)$ we have

$$
\begin{aligned}
& { }_{2} F_{1}\left(\chi_{1}, \chi_{2}, \chi_{3} ; 1-\mathrm{e}^{-\alpha r}\right)= \\
& \Gamma\left(\chi_{3}\right)\left[\frac{\Gamma\left(\chi_{3}-\chi_{1}-\chi_{2}\right)}{\Gamma\left(\chi_{3}-\chi_{1}\right) \Gamma\left(\chi_{3}-\chi_{2}\right)}\right. \\
& \left.+\mathrm{e}^{-2 \mathrm{i} k r}\left(\frac{\Gamma\left(\chi_{3}-\chi_{1}-\chi_{2}\right)}{\Gamma\left(\chi_{3}-\chi_{1}\right) \Gamma\left(\chi_{3}-\chi_{2}\right)}\right)^{*}\right],
\end{aligned}
$$

where we have used the following relation:

$$
\begin{aligned}
& \chi_{3}-\chi_{1}-\chi_{2}=\frac{2 \mathrm{i} k}{\alpha}=\left(\chi_{1}+\chi_{2}-\chi_{3}\right)^{*}, \\
& \chi_{3}-\chi_{1}=\chi_{2}^{*}, \\
& \chi_{3}-\chi_{2}=\chi_{1}^{*} .
\end{aligned}
$$

Therefore the corresponding unnormalized wave functions for $r \rightarrow \infty(s \rightarrow 1)$ are given as

$$
\begin{aligned}
& G_{n \kappa}^{p s}(r \rightarrow \infty)=N_{n \kappa}^{p s} \Gamma\left(\chi_{3}\right)\left[\mathrm{e}^{\mathrm{i} k r} \frac{\Gamma\left(\chi_{3}-\chi_{1}-\chi_{2}\right)}{\Gamma\left(\chi_{3}-\chi_{1}\right) \Gamma\left(\chi_{3}-\chi_{2}\right)}\right. \\
& \left.+\mathrm{e}^{-\mathrm{i} k r}\left(\frac{\Gamma\left(\chi_{3}-\chi_{1}-\chi_{2}\right)}{\Gamma\left(\chi_{3}-\chi_{1}\right) \Gamma\left(\chi_{3}-\chi_{2}\right)}\right)^{*}\right] .
\end{aligned}
$$

Introducing

$\frac{\Gamma\left(\chi_{3}-\chi_{1}-\chi_{2}\right)}{\Gamma\left(\chi_{3}-\chi_{1}\right) \Gamma\left(\chi_{3}-\chi_{2}\right)}=\left|\frac{\Gamma\left(\chi_{3}-\chi_{1}-\chi_{2}\right)}{\Gamma\left(\chi_{3}-\chi_{1}\right) \Gamma\left(\chi_{3}-\chi_{2}\right)}\right| \mathrm{e}^{\mathrm{i} \delta_{p s}}$ allows us to obtain

$$
\begin{aligned}
& G_{n \kappa}^{p s}(r)=2 N_{n \kappa}^{p s} \Gamma\left(\chi_{3}\right)\left|\frac{\Gamma\left(\chi_{3}-\chi_{1}-\chi_{2}\right)}{\Gamma\left(\chi_{3}-\chi_{1}\right) \Gamma\left(\chi_{3}-\chi_{2}\right)}\right| \\
& \quad \times \sin \left(k r+\frac{\pi}{2}+\delta_{p s}\right) .
\end{aligned}
$$

By comparing Eq. (30) with the boundary condition [26-28] $r \rightarrow \infty \Rightarrow G_{n \kappa}^{p s}(\infty) \rightarrow 2 \sin \left(k r-\frac{\kappa \pi}{2}+\delta_{\kappa}^{p s}\right)$ phase shifts and the normalized constant can be given by

$$
\begin{aligned}
& \delta_{\kappa}^{p s}=(\kappa+1) \frac{\pi}{2}+\delta_{p s}=(\kappa+1) \frac{\pi}{2}+ \\
& \times \arg \left(\frac{\Gamma\left(\frac{2 \mathrm{i} k}{\alpha}\right)}{\Gamma\left(\eta_{\kappa}+\frac{\mathrm{i} k}{\alpha}-\sqrt{\Lambda_{3}}\right) \Gamma\left(\eta_{\kappa}+\frac{\mathrm{i} k}{\alpha}+\sqrt{\Lambda_{3}}\right)}\right), \\
& N_{n \kappa}^{p s}=\frac{1}{\Gamma\left(2 \eta_{\kappa}\right)} \\
& \times\left|\frac{\Gamma\left(\eta_{\kappa}+\frac{\mathrm{i} k}{\alpha}-\sqrt{\Lambda_{3}}\right) \Gamma\left(\eta_{\kappa}+\frac{\mathrm{i} k}{\alpha}+\sqrt{\Lambda_{3}}\right)}{\Gamma\left(\frac{2 \mathrm{i} k}{\alpha}\right)}\right| .
\end{aligned}
$$

\subsection{Spin symmetry limit}

In the spin symmetry limit condition, we take the sum potential $\Sigma(r)$ as the Hellmann potential, the difference potential $\Delta(r)$ as constant and the tensor potential $U(r)$ as the Coulomb-like interaction term. Thus, we have the following:

$$
\begin{aligned}
& \Sigma(r)=-\frac{a^{s}}{r}+b^{s} \frac{\mathrm{e}^{-\alpha r}}{r}, \quad \Delta(r)=C_{s}, \\
& U(r)=-\frac{H}{r} .
\end{aligned}
$$

Substituting Eqs. (33) and (34) into Eq. (11) and making two transformation i.e., $s=1-\mathrm{e}^{-\alpha r}$ and $F_{n \kappa}^{s}(s)=$ $s^{\gamma^{\prime}}(1-s)^{\beta^{\prime}} f_{n \kappa}^{s}(s)$ yields

$$
\begin{aligned}
& {\left[s(1-s) \frac{\mathrm{d}^{2}}{\mathrm{~d} s^{2}}+\left(\chi_{3}^{\prime}-\left(1+\chi_{1}^{\prime}+\chi_{2}^{\prime}\right) s\right) \frac{\mathrm{d}}{\mathrm{d} s}-\chi_{1}^{\prime} \chi_{2}^{\prime}\right]} \\
& \quad \times f_{n \kappa}^{s}(s)=0,
\end{aligned}
$$

where

$$
\begin{aligned}
& \chi_{1}^{\prime}=\gamma^{\prime}+\beta^{\prime}+\sqrt{\Lambda_{3}^{\prime}}, \\
& \chi_{2}^{\prime}=\gamma^{\prime}+\beta^{\prime}-\sqrt{\Lambda_{3}^{\prime}}, \\
& \chi_{3}^{\prime}=2 \gamma^{\prime}, \\
& \gamma^{\prime}=\Lambda_{\kappa}, \\
& \beta^{\prime}=-\frac{i k^{\prime}}{\alpha}, \quad k^{\prime}=\sqrt{\alpha^{2} \Lambda_{2}^{\prime}} .
\end{aligned}
$$

and

$\Lambda_{1}^{\prime}, \Lambda_{2}^{\prime}, \Lambda_{3}^{\prime}$ and $\Lambda_{\kappa}$ are defined as

$$
\begin{aligned}
& \Lambda_{1}^{\prime}=-\Lambda_{\kappa}\left(\Lambda_{\kappa}-1\right), \\
& \Lambda_{2}^{\prime}=-\Lambda_{\kappa}\left(\Lambda_{\kappa}-1\right)-\frac{1}{\alpha^{2}}\left(M-E_{n, \kappa}^{s}\right) \\
& \quad\left(M+E_{n, \kappa}^{s}-C_{s}\right)+\frac{a^{s}}{\alpha}\left(M+E_{n, \kappa}^{s}-C_{s}\right), \\
& \Lambda_{3}^{\prime}=\frac{1}{\alpha^{2}}\left(M-E_{n, \kappa}^{s}\right)\left(M+E_{n, \kappa}^{s}-C_{s}\right) \\
& \quad-\frac{b^{s}}{\alpha}\left(M+E_{n, \kappa}^{s}-C_{s}\right),
\end{aligned}
$$$$
\Lambda_{\kappa}=\kappa+H+1 \text {. }
$$

The solution of Eq. (35) can be expressed in hypergeometric functions as

$$
f_{n \kappa}^{s}(s)={ }_{2} F_{1}\left(\chi_{1}^{\prime}, \chi_{2}^{\prime}, \chi_{3}^{\prime} ; s\right) .
$$

By doing the same approach as the previous subsection, the phase shifts and the normalized constant of spin symmetry limit can be given by

$$
\begin{gathered}
\delta_{\kappa}^{s}=(\kappa+1) \frac{\pi}{2}+\delta_{s}=(\kappa+1) \frac{\pi}{2} \\
+\arg \left(\frac{\Gamma\left(\frac{2 \mathrm{i} k^{\prime}}{\alpha}\right)}{\Gamma\left(\Lambda_{\kappa}+\frac{\mathrm{i} k^{\prime}}{\alpha}-\sqrt{\Lambda_{3}^{\prime}}\right) \Gamma\left(\Lambda_{\kappa}+\frac{\mathrm{i} k^{\prime}}{\alpha}+\sqrt{\Lambda_{3}^{\prime}}\right)}\right) \\
N_{n \kappa}^{s}=\frac{1}{\Gamma\left(2 \Lambda_{\kappa}\right)} \\
\times\left|\frac{\Gamma\left(\Lambda_{\kappa}+\frac{\mathrm{i} k^{\prime}}{\alpha}-\sqrt{\Lambda_{3}^{\prime}}\right) \Gamma\left(\Lambda_{\kappa}+\frac{\mathrm{i} k^{\prime}}{\alpha}+\sqrt{\Lambda_{3}^{\prime}}\right)}{\Gamma\left(\frac{2 \mathrm{i} k^{\prime}}{\alpha}\right)}\right|
\end{gathered}
$$




\section{Result and discussions}

In this section, we will investigate scattering states of Yukawa-like and Coulomb-like potentials as special cases of the Hellmann potential.

\subsection{Yukawa-like scattering states}

By considering $a^{p s}=0\left(a^{s}=0\right)$, the Hellmann potential changes into the Yukawa potential. According to our results for the pseudo spin symmetry we have

$$
\begin{aligned}
& \delta_{\kappa}^{p s}=(\kappa+1) \frac{\pi}{2}+\delta_{p s}= \\
& \quad(\kappa+1) \frac{\pi}{2}+\arg \left(A_{1}\right)-\arg \left(A_{2} A_{3}\right), \\
& N_{n \kappa}^{p s}=\frac{1}{\Gamma\left(2 \eta_{\kappa}\right)\left|A_{1}\right|}\left|A_{2}\right|\left|A_{3}\right|,
\end{aligned}
$$

where $A_{1}=\Gamma\left(2 \mathrm{i}\left[-\eta_{\kappa}\left(\eta_{\kappa}-1\right)-\frac{1}{\alpha^{2}}\left(M+E_{n, \kappa}^{p s}\right)\right.\right.$ $\left.\left.\times\left(M-E_{n, \kappa}^{p s}+C_{p s}\right)\right]^{1 / 2}\right), A_{2}=\Gamma\left(\eta_{\kappa}+\mathrm{i}\left[-\eta_{\kappa}\left(\eta_{\kappa}-1\right)\right.\right.$ $\left.\left.-\frac{1}{\alpha^{2}}\left(M+E_{n, \kappa}^{p s}\right)\left(M-E_{n, \kappa}^{p s}+C_{p s}\right)\right]^{1 / 2}-\sqrt{\Lambda_{3}}\right), A_{3}=\Gamma\left(\eta_{\kappa}\right.$ $+\mathrm{i}\left[-\eta_{\kappa}\left(\eta_{\kappa}-1\right)-\frac{1}{\alpha^{2}}\left(M+E_{n, \kappa}^{p s}\right)\left(M-E_{n, \kappa}^{p s}+C_{p s}\right)\right]^{1 / 2}$ $+\sqrt{\Lambda_{3}}$ ) and from Eq. (41) and Eq. (42) for the spin symmetry limit one can find

$$
\begin{aligned}
& \delta_{\kappa}^{s}=(\kappa+1) \frac{\pi}{2}+\delta_{s}=(\kappa+1) \frac{\pi}{2}+\arg \left(\frac{A_{4}}{A_{5} A_{6}}\right), \\
& N_{n \kappa}^{s}=\frac{1}{\Gamma\left(2 \Lambda_{\kappa}\right)}\left|\frac{A_{5} A_{6}}{A_{4}}\right| .
\end{aligned}
$$

where $A_{4}=\Gamma\left(2 \mathrm{i}\left[-\Lambda_{\kappa}\left(\Lambda_{\kappa}-1\right)-\frac{1}{\alpha^{2}}\left(M+E_{n, \kappa}^{p s}\right)\right.\right.$ $\left.\left.\left(M-E_{n, \kappa}^{p s}+C_{p s}\right)\right]^{1 / 2}\right), A_{5}=\Gamma\left(\Lambda_{\kappa}+\mathrm{i}\left[-\Lambda_{\kappa}\left(\Lambda_{\kappa}-1\right)\right.\right.$ $\left.\left.-\frac{1}{\alpha^{2}}\left(M+E_{n, \kappa}^{p s}\right)\left(M-E_{n, \kappa}^{p s}+C_{p s}\right)\right]^{1 / 2}-\sqrt{\Lambda_{3}^{\prime}}\right)$, $A_{6}=\Gamma\left(\Lambda_{\kappa}+\mathrm{i}\left[-\Lambda_{\kappa}\left(\Lambda_{\kappa}-1\right)-\frac{1}{\alpha^{2}}\left(M+E_{n, \kappa}^{p s}\right) \times\right.\right.$ $\left.\left.\left(M-E_{n, \kappa}^{p s}+C_{p s}\right)\right]^{1 / 2}+\sqrt{\Lambda_{3}^{\prime}}\right)$.

\subsection{Coulomb-like scattering states}

If we choose $b^{p s}=0\left(b^{s}=0\right)$ the Hellmann potential reduces to the Coulomb-like potential. In this case Eq. (15) changes into

$$
\left[\frac{\mathrm{d}^{2}}{\mathrm{~d} r^{2}}+k^{2}+\frac{2 \lambda}{r}-\frac{l^{\prime}\left(l^{\prime}+1\right)}{r^{2}}\right] G_{n, \kappa}^{p s}(r)=0,
$$

where $l^{\prime}=\frac{1}{2}\left[-1 \pm\left(1-2 \eta_{\kappa}\right)\right]$, $k=\sqrt{\left(M+E_{n, \kappa}^{p s}\right)\left(-M+E_{n, \kappa}^{p s}-C_{p s}\right)}$,

$\lambda=-\frac{a^{p s}}{2}\left(M-E_{n, \kappa}^{p s}+C_{p s}\right)$.

Considering the boundary conditions of the scattering states, i.e. $G_{n, \kappa}^{p s}(r=0)=0$, we take the following ansatz for the asymptotic behavior of the wave function at the origin:

$$
G_{n, \kappa}^{p s}(r)=A(k r)^{l^{\prime}+1} \mathrm{e}^{\mathrm{i} k r} f(r) .
$$

Inserting Eq. (48) into Eq. (47), we obtain

$$
r \frac{\mathrm{d}^{2} f}{\mathrm{~d} r^{2}}+\left(2 l^{\prime}+2+2 \mathrm{i} k r\right) \frac{\mathrm{d} f}{\mathrm{~d} r}+\left[2 \lambda+2 \mathrm{i} k\left(l^{\prime}+1\right)\right] f(r)=0 .
$$

$$
z \frac{\mathrm{d}^{2} f}{\mathrm{~d} z^{2}}+\left(2 l^{\prime}+2-z\right) \frac{\mathrm{d} f}{\mathrm{~d} z}-\left[l^{\prime}+1-\frac{\mathrm{i} \lambda}{k}\right] f(z)=0 .
$$

Eq. (50) is just the confluent hypergeometric equation and its solutions are the confluent hypergeometric functions. The analytical solution as $r \rightarrow 0$ is

$$
f(r)=F\left(l^{\prime}+1-\frac{\mathrm{i} \lambda}{k}, 2 l^{\prime}+2,-2 \mathrm{i} k r\right) .
$$

Thus, the radial wave function of the scattering states is $G_{n, \kappa}^{p s}(r)=A(k r)^{l^{\prime}+1} \mathrm{e}^{\mathrm{i} k r} F\left(l^{\prime}+1-\frac{\mathrm{i} \lambda}{k}, 2 l^{\prime}+2,-2 \mathrm{i} k r\right)$

Now, we want to obtain the asymptotic behavior of wave function for $r \rightarrow \infty$ and then calculate the normalization constant and the phase shifts. To proceed on, we use the transformation formulae for confluent hypergeometric functions when $z \rightarrow \infty$ :

$$
F(\alpha, \gamma, z)=\frac{\Gamma(\gamma)}{\Gamma(\alpha)} \mathrm{e}^{z} z^{\alpha-\gamma}+\frac{\Gamma(\gamma)}{\Gamma(\gamma-\alpha)} \mathrm{e}^{ \pm \mathrm{i} \pi \alpha} z^{-\alpha},
$$

where "+" corresponds to $-\pi / 2<\arg (z)<3 \pi / 2$, and "-" corresponds to $-3 \pi / 2<\arg (z)<\pi / 2$. Equation (53) is then changed into

$$
\begin{aligned}
& F\left(l^{\prime}+1-\frac{\mathrm{i} \lambda}{k}, 2 l^{\prime}+2,-2 \mathrm{i} k r\right)= \\
& \quad \frac{\Gamma\left(2 l^{\prime}+2\right)}{\Gamma\left(l^{\prime}+1-\frac{\mathrm{i} \lambda}{k}\right)} \mathrm{e}^{-2 \mathrm{i} k r}(2 k r)^{-\left(l^{\prime}+1+\frac{\mathrm{i} \lambda}{k}\right)} \mathrm{e}^{\mathrm{i}\left(l^{\prime}+1+\frac{\mathrm{i} \lambda}{k}\right) \frac{\pi}{2}} \\
& \quad+\frac{\Gamma\left(2 l^{\prime}+2\right)}{\Gamma\left(l^{\prime}+1+\frac{\mathrm{i} \lambda}{k}\right)}(2 k r)^{-\left(l^{\prime}+1-\frac{\mathrm{i} \lambda}{k}\right)} \mathrm{e}^{-\mathrm{i}\left(l^{\prime}+1-\frac{\mathrm{i} \lambda}{k}\right) \frac{\pi}{2}},(5
\end{aligned}
$$

where

$$
z=-2 \mathrm{i} k r=|z| \mathrm{e}^{-\mathrm{i} \frac{\pi}{2}} .
$$

Letting

$$
\begin{aligned}
& \Gamma\left(l^{\prime}+1-\frac{\mathrm{i} \lambda}{k}\right)=\left|\Gamma\left(l^{\prime}+1-\frac{\mathrm{i} \lambda}{k}\right)\right| \mathrm{e}^{\mathrm{i} \delta_{p s},} \\
& \Gamma\left(l^{\prime}+1+\frac{\mathrm{i} \lambda}{k}\right)=\left|\Gamma\left(l^{\prime}+1-\frac{\mathrm{i} \lambda}{k}\right)\right| \mathrm{e}^{-\mathrm{i} \delta_{p s},}
\end{aligned}
$$

Eq. (56) can be written as

$$
\begin{aligned}
& F\left(l^{\prime}+1-\frac{\mathrm{i} \lambda}{k}, 2 l^{\prime}+2,-2 \mathrm{i} k r\right)= \\
& \quad \frac{\Gamma\left(2 l^{\prime}+2\right)}{\left|\Gamma\left(l^{\prime}+1-\frac{\mathrm{i} \lambda}{k}\right)\right|} \frac{\exp \left(-\frac{\pi \lambda}{2 k}-\mathrm{i} k r\right)}{(2 k r)\left(l^{\prime}+1\right)} \\
& \quad \times\left(\exp \left(\frac{\mathrm{i} \lambda}{k} \ln (2 k r)-\mathrm{i} \delta_{p s}+\mathrm{i}\left(l^{\prime}+1\right) \frac{\pi}{2}\right)\right. \\
& \left.\quad+\exp \left(\frac{\mathrm{i} \lambda}{k} \ln (2 k r)+\mathrm{i} \delta_{p s}-\mathrm{i}\left(l^{\prime}+1\right) \frac{\pi}{2}\right)\right)= \\
& \quad \frac{\Gamma\left(2 l^{\prime}+2\right)}{\left|\Gamma\left(l^{\prime}+1-\frac{\mathrm{i} \lambda}{k}\right)\right|} \frac{\exp \left(-\frac{\pi \lambda}{2 k}-\mathrm{i} k r\right)}{(2 k r)^{\left(l^{\prime}+1\right)}} \\
& \quad \times 2 \sin \left(k r+\delta_{p s}-\frac{\pi l^{\prime}}{2}+\frac{\lambda}{k} \ln (2 k r)\right) .
\end{aligned}
$$

By putting Eq. (57) into Eq. (52), we have

$$
\begin{aligned}
& G_{n, \kappa}^{p s}(r) \stackrel{(r \rightarrow \infty)}{\longrightarrow} \frac{A \Gamma\left(2 l^{\prime}+2\right)}{\left|\Gamma\left(l^{\prime}+1-\frac{\mathrm{i} \lambda}{k}\right)\right|} \frac{\mathrm{e}^{-\frac{\pi \lambda}{2 k}}}{2^{\left(l^{\prime}+1\right)}} \\
& \quad \times 2 \sin \left(k r+\delta_{p s}-\frac{\pi l^{\prime}}{2}+\frac{\lambda}{k} \ln (2 k r)\right) .
\end{aligned}
$$

Introducing $z=-2 \mathrm{i} k r$, Eq. (49) can be modified as 
On the other hand,

$G_{n, \kappa}^{p s}(r \rightarrow \infty)=2 \sin \left(k r+\delta_{\kappa}^{p s}-\frac{\kappa \pi}{2}+\frac{\lambda}{k} \ln (2 k r)\right)$.

Therefore phase shifts and the normalization constant can be found as

$$
\begin{gathered}
\delta_{\kappa}^{p s}=\frac{\kappa \pi}{2}+\delta_{p s}-\frac{\pi l^{\prime}}{2}=\frac{\pi}{2}\left(\kappa-l^{\prime}\right) \\
+\arg \left(\Gamma\left(l^{\prime}+1-\frac{\mathrm{i} \lambda}{k}\right)\right), \\
A=\frac{\mathrm{e}^{\frac{\pi \lambda}{2 k}}\left|\Gamma\left(l^{\prime}+1-\frac{\mathrm{i} \lambda}{k}\right)\right| 2^{\left(l^{\prime}+1\right)}}{\Gamma\left(2 l^{\prime}+2\right)} .
\end{gathered}
$$

By doing the same approach as for pseudospin symmetry, we obtain phase shifts and the normalization constant of spin symmetry as follows:

$$
\begin{gathered}
\delta_{\kappa}^{s}=\frac{\kappa \pi}{2}+\delta_{s}-\frac{\pi l^{\prime}}{2}=\frac{\pi}{2}\left(\kappa-l^{\prime}\right) \\
+\arg \left(\Gamma\left(l^{\prime}+1-\frac{\mathrm{i} \lambda}{k}\right)\right), \\
A=\frac{\mathrm{e}^{\frac{\pi \lambda}{2 k}}\left|\Gamma\left(l^{\prime}+1-\frac{\mathrm{i} \lambda}{k}\right)\right| 2^{\left(l^{\prime}+1\right)}}{\Gamma\left(2 l^{\prime}+2\right)},
\end{gathered}
$$

where in the spin symmetry case $l^{\prime}, k$, and $\lambda$ are defined as:

$$
\begin{aligned}
l^{\prime} & =\frac{1}{2}\left[-1 \pm\left(1-2 \Lambda_{\kappa}\right)\right], \\
k & =\sqrt{\left(-M+E_{n, \kappa}^{s}\right)\left(M+E_{n, \kappa}^{s}-C_{s}\right)}, \\
\lambda & =\frac{a^{s}}{2}\left(M+E_{n, \kappa}^{s}-C_{s}\right) .
\end{aligned}
$$

\section{Conclusions}

We have analyzed the characteristics of relativistic scattering states of the Dirac equation with the Hellmann potential for both spin and pseudo spin symmetries. Due to the centrifugal term we have used a proper approximation, and obtained approximately the normalized wave function and scattering phase shifts for both symmetries. Also, we have studied two special cases of the Hellmann potential, i.e., the Yukawa-like and Coulomb-like potentials.

\section{Acknowledgments}

We wish to give our sincere gratitude to the referees for their instructive comments and careful reading of the article.

\section{References}

[1] J.N. Ginocchio, Phys. Rep. 414, 165 (2005).

[2] P.R. Page, T. Goldman, J.N. Ginocchio, Phys. Rev Lett. 86, 204 (2001).

[3] K.T. Hecht, A. Adler, Nucl. Phys. A 137, 129 (1969).

[4] A. Arima, M. Harvey, K. Shimizu, Phys. Lett. B 30, 517 (1969).

[5] G.F. Wei, S.H. Dong, Eur. Phys. J. A 43, 185 (2010).

[6] R. Lisboa, M. Malheiro, P. Alberto, M. Fiolhais, A.S. de Castro, Phys. Rev. C 81, 064324 (2010).

[7] H. Hassanabadi, B.H. Yazarloo, M. Mahmoudieh, S. Zarrinkamar, Eur. Phys. J. Plus 128, 111 (2013).

[8] P. Alberto, A.S. de Castro, M. Malheiro, Phys. Rev. C 87, 031301(R) (2013).

[9] G.F. Wei, S.H. Dong, Phys. Lett. B 686, 288 (2010).

[10] H. Hassanabadi, B.H. Yazarloo, N. Salehi, Indian J. Phys. 88, 405 (2014).

[11] C.S. Jia, J.Y. Liu, P.Q. Wang, X. Lin, Int. J. Theor. Phys. 48, 2633 (2009).

[12] C.S. Jia, P.Q. Wang, J.Y. Liu, S. He, Int. J. Theor Phys. 47, 2513 (2008).

[13] C.S. Jia, Y.F. Diao, J.Y. Liu, Int. J. Theor. Phys. 47, 664 (2008).

[14] G.F. Wei, S.H. Dong, Europhys. Lett. 87, 40004 (2009).

[15] H. Hassanabadi, S. Sargolzaeipor, B.H. Yazarloo, Few-Body Syst. 56, 115 (2015).

[16] G.F. Wei, S.H. Dong, Phys. Lett. A 373, 49 (2008).

[17] G.F. Wei, S.H. Dong, Phys. Lett. A 373, 2428 (2009).

[18] G.F. Wei, S.H. Dong, Phys. Scr. 81, 035009 (2010).

[19] H. Hellmann, Acta Physicochim. URSS 1, 913 (1935).

[20] J. Adamowski, Phys. Rev. A 31, 43 (1985).

[21] R. Dutt, U. Mukherji, Y.P. Varshni, Phys. Rev. A 34, 777 (1986).

[22] S. Bednarek, J. Adamowski, M. Suffczyński, Solid State Commun. 21, 1 (1977).

[23] G.F. Wei, S.H. Dong, Eur. Phys. J. A 46, 207 (2010).

[24] R.L. Greene, C. Aldrich, Phys. Rev. A 14, 2363 (1976).

[25] W.C. Qiang, R.S. Zhou, Y. Gao, Phys. Lett. A 371, 201 (2007).

[26] L.D. Landau, E.M. Lifshitz, Quantum Mechanics, Non-Relativistic Theory, 3rd ed., Pergamon, New York 1977.

[27] H. Hassanabadi, B.H. Yazarloo, Indian J. Phys. 87, 1017 (2013).

[28] B.H. Yazarloo, L.L. Lu, G. Liu, S. Zarrinkamar, H. Hassanabadi, Adv. High En. Phys. 2013, 317605 (2013). 